\title{
From the National Institute of Allergy and Infectious Diseases
}

\section{Summary of the 21st United States-Japan Joint Cholera Conference}

\author{
Robert Edelman and R. Bradley Sack
}

From the Microbiology and Infectious Diseases Program, National Institute of Allergy and Infectious Diseases, National Institutes of Health, Bethesda; and the Division of Geographic Medicine, School of Hygiene and Public Health, Johns Hopkins University, Baltimore, Maryland

The 21st Joint Conference on Cholera, sponsored by the United States-Japan Cooperative Medical Science Program and the Microbiology and Infectious Diseases Program of the National Institute of Allergy and Infectious Diseases, was held at the $\mathrm{Na}$ tional Institutes of Health (Bethesda, Md) on 21-23 October 1985. This conference, which is held annually in either the United States or Japan, provides a forum for international communication and collaboration by investigators working on enterotoxigenic bacterial infections. More than 148 individuals from the United States and five foreign countries attended the conference. Four members of the Japanese Cholera Panel and 10 additional Japanese guests participated. Thirty-six papers were chosen for presentation from among 48 submitted abstracts. The program consisted of the following sections: epidemiology and clinical studies, bacteriology and virulence factors, toxinology and genetics, immunol-

Participants in the conference included the following: P. Blake, Centers for Disease Control, Allanta; J. D. Clemens, International Centre for Diarrhoeal Disease Research, Dhaka, Bangladesh; J. D. Clements, Tulane University Medical Center, New Orleans; M. B. Cohen, University of Cincinnati Medical Center, Cincinnati; R. R. Colwell, University of Maryland, College Park, Maryland; R. Edelman, National Institute of Allergy and Infectious Diseases, Bethesda, Maryland; R. A. Finkelstein, University of Missouri, Columbia, Missouri; A. Ghoda, Kitasato University, Tokyo; R. L. Guerrant, University of Virginia, Charlottesville, Virginia; T. Hirayama, University of Tokyo, Tokyo; K. Hisatsune, Josai University, Saitama, Japan; T. Honda, Osaka University, Osaka, Japan; T. Itoh, Juntendo University, Tokyo; M. Iwanaga, University of the Ryukyu, Okinawa, Japan; Y. Kudoh, Tokyo Metropolitan Research Laboratory of Public Health, Tokyo; Y. M. Kupersztoch, University of Texas Health Science Center, Dallas; S. Kuwahara, Toho University, Tokyo; M. M. Levine, University of Maryland, Baltimore; J. J. Mathewson, University of Texas Health Science Center, Houston; J. J. Mekalanos, Harvard Medical School, Boston; H. Moon, National Animal Diseases Laboratory, Ames, lowa; J. Moss, National Heart, Lung, and Blood Institute, Bethesda, Maryland; J. R. Murphy, Boston University Medical Center, Boston; J. P. Nataro, University of Maryland, Baltimore; J. Newland, Uniformed Services University of the ogy and vaccine development, and pathophysiology. Selected papers from each of the program sections are summarized below.

\section{Epidemiology and Clinical Studies}

Dr. M. Santosham described prospective studies of the incidences and etiologies of diarrhea on the Fort Apache Indian Reservation in Arizona for a threeyear period beginning in April 1981. Not unexpectedly, the highest attack rate of diarrhea was found in children less than two years old, with four to five episodes per year in children during this time - a rate similar to that in many developing countries. A viral pathogen, either rotavirus, coronavirus, or adenovirus, was most frequently found in infants less than one year of age. Rotavirus and Shigella were the most common pathogens found in hospitalized patients and in outpatients, respectively.

Health Sciences, Bethesda, Maryland; N. Ohtomo, The ChemoSero-Therapeutics Research Institute, Kumamoto, Japan; S. H. Richardson, Wake Forest University, Winston-Salem, North Carolina; D. C. Robertson, University of Kansas, Lawrence, Kansas; R. B. Sack, Johns Hopkins University, Baltimore; E. SalazarLindo, Universidad Peruana Cayetano Heredia, Lima, Peru; M. Santosham, Johns Hopkins University, Whiteriver, Arizona; C. V. Sciortino, Veterans Administration, Charleston, South Carolina; T. Shimamura, Tokai University, Kanagawa, Japan; Y. Shimonishi, Osaka University, Osaka, Japan; N. Strockbine, Uniformed Services University of the Health Sciences, Bethesda, Maryland; $Y$. Takeda, University of Tokyo, Tokyo; M. R. Thompson, University of Cincinnati Medical Center, Cincinnati; M. Tomisato, University of the Ryukyu, Okinawa, Japan; H. Watanabe, National Institute of Health, Tokyo; C. S. Weikel, University of Virginia, Charlottesville, Virginia; S. C. Whipp, National Animal Disease Center, Ames, Iowa; T. Yokota, Juntendo University, Tokyo; and Y. Zinnaka, National Defense Medical College, Saitama, Japan.

Please address requests for reprints to Dr. Robert Edelman, Building 31, Room 7A49, National Institute of Allergy and Infectious Diseases, National Institutes of Health, Bethesda, Maryland 20892. 
A 1984 cholera epidemic in Mali was reported by Dr. P. A. Blake. Multiple routes of transmission, including contaminated wells and stagnant millet broth, accounted for a mean cohort attack rate of $1.5 \%$. Lack of use of rehydration therapy on the part of patients and health care personnel alike, together with maldistribution of oral rehydration solutions, accounted for a case-fatality rate of $\mathbf{3 0 \%}$.

J. J. Mathewson measured the prevalence of HEp2 cell-adherent Escherichia coli (EAEC) among American travelers to Mexico. Only four of 42 EAEC strains belonged to traditional enteropathogenic E. coli (EPEC) O serogroups, and none secreted known enterotoxins. EAEC were recovered almost twice as often in patients with diarrhea as in well individuals. Overall, EAEC were associated with $30 \%$ of diarrheal episodes having no other identifiable etiologic cause. About half of the patients with EAEC had a serum antibody response to the strain isolated from their stool specimens. Some volunteers challenged orally with selected EAEC strains suffered bowel infection and diarrheal illness. Thus, EAEC are likely causes of some cases of travelers' diarrhea.

Dr. Y. Kudoh reported an outbreak of acute enteritis in an elementary school in Tokyo that was caused by $E$. coli strain $\mathrm{O} 145: \mathrm{H}^{-}$. This strain produced a poorly characterized toxin that was biologically similar to Shiga toxin but immunologically distinct from Shiga toxin, LT (labile toxin), and ST (stable toxin).

Dr. R. Colwell grew Vibrio cholerae $\mathrm{O} 1$ in microcosms simulating conditions of the estuarine environment. Dr. Colwell discovered that under these in vitro conditions, the bacteria can enter into and persist in a dormant state, refractory to culture by routine laboratory methods. The bacteria, however, were recovered by animal passage, and they retained the potential for virulence. Dr. Colwell suggested that such cryptic $V$. cholerae may exist in nature.

Dr. E. Salazar-Lindo reported a double-blind, placebo-controlled trial of erythromycin treatment of dysentery in Peruvian children aged three to 60 months. Treatment was started immediately, before culture results were known, and always within five days of onset of dysentery. In campylobacter disease, erythromycin resulted in a significant shortening of both clinical illness and excretion of $C$. jejuni compared with placebo; however, erythromycin treatment failed to affect the illness caused by Shigella organisms.

\section{Bacteriology and Virulence Factors}

Monoclonal antibodies have helped to identify and purify virulence antigens of $V$. cholerae. The identification of 16 separate outer-membrane antigens of $V$. chloerae has been accomplished by C. V. Sciortino by using a library of 66 monoclonal antibodies. One monoclonal antibody, combined with an outer-membrane protein identified as an ironregulated porin, killed $\boldsymbol{V}$. cholerae in the absence of complement. Using a monoclonal antibody, $\mathbf{M}$. Tomisato has identified an antigenically distinct pililike adhesin on $V$. cholerae Ogawa strains, but not on Inaba strains.

Dr. H. Hisatsune analyzed the sugars of the polysaccharide portions of LPS contained in non-O1 group V. cholerae, Vibrio fluvialis, and Vibrio vulnificus and related these sugars to the chemotaxonomy of these organisms. T. Ito reported that cyclic AMP added to cultures of $V$. cholerae modulated the growth and physiology of 1940 and 1982 isolates differently. Drs. M. Iwanaga and T. Shimamura modulated production of cholera toxin in vitro by changing bicarbonate and atmospheric $\mathrm{CO}_{2}$ concentrations, by adding or deleting thiol compounds to the growth media, and by immobilizing culture flasks for several hours before shaking them.

Dr. J. Moss reported experiments establishing that intestinal epithelial cells contain an ADP-ribosylarginine cleavage enzyme. This discovery raises the possibility that such cells may have the ability to reverse the cholera toxin-catalyzed ADP-ribosylation reaction and, thus, the metabolic derangements secondary to adenylate cyclase activation. The ADPribosylarginine cleavage enzyme could thus assist in recovery from cholera.

Dr. D. C. Robertson summarized recent results of his long-term studies of the synthesis and cellular distribution of heat-labile enterotoxigenic $E$. coli (ETEC) enterotoxins. LT subunits A and B are localized in the periplasmic space of ETEC, whereas cholera toxin is actively exported from $V$. cholerae. The question of how LT leaves ETEC is yet unanswered; possibilities include passive leakage or lysis of the cell. Dr. J. Mekalanos, by using a recently developed set of fusion vectors for genetic analysis of extracellularly secreted proteins of $V$. cholerae, postulated that the A and B subunits of cholera toxin are assembled in the periplasmic space before they are secreted into the extracellular environment. 


\section{Toxinology and Genetics}

Dr. N. A. Strockbine discovered that $E$. coli strain O157:H7, which causes epidemic hemorrhagic colitis, harbors two different cytotoxin-converting phages. Each of these phages contain a gene coding for a cytotoxin with Shiga-like biologic properties but with different antigenic properties. Shiga antitoxin neutralizes one toxin, but not the other. Characterization of the new, non-Shiga toxin is underway. Cloning and DNA sequencing of the Shiga-like toxin gene to determine the primary structure of its product was reported by Dr. J. W. Newland.

Dr. J. P. Nataro described genetic analyses of EPEC virulence plasmids. He discovered that full virulence of many EPEC serotypes appears to be dependent upon a 55-65-megadalton plasmid that encodes adherence to HEp-2 cells, a 94-kilodalton protein, and possibly other functional proteins. Early results from volunteer studies reveal the 94-kilodalton protein is immunogenic, is associated with EPEC virulence, and, possibly, is an intestinal adhesion factor for EPEC.

Detailed genetic analysis of the cyclic AMP-mediated transcriptional regulation of the ST-1 enterotoxin of $E$. coli strains was reported by Y. M. Kupersztoch. He postulated the existence of a protease that nick translates ST in the periplasmic space, an occurence allowing the ST to be exported from the cell.

A 120-140-megadalton plasmid of Shigella spp. encodes functions necessary for invasion of epithelial cells. Dr. H. Watanabe devised a method to transfer these nonconjugative plasmids from virulent Shigella into nonvirulent E. coli and Shigella. He examined the restoration of invasiveness in the strain that received the plasmid or plasmid fragments. A 4.1-kilobase DNA sequence, conserved among Shigella spp., is necessary for invasions.

Microbial toxins act through cyclic AMP, cyclic GMP, or poorly understood calcium-dependent, cyclic nucleotide-independent pathways to cause intestinal secretion. Dr. C. S. Weikel described studies of the latter pathway that involved activation of protein kinase $\mathrm{C}$ (PKC) in piglet jejunum. Dr. Weikel showed that phorbol esters, which directly activate $\mathrm{PKC}$, cause net water and electrolyte secretion detectable in vivo within $30 \mathrm{~min}$ in piglet jejunum; this secretion persists for several hours. These important results suggest a direct role for PKC in intestinal secretion.
A series of papers focused on STs. Dr. T. Hirayama confirmed previous reports that cholera and STs stimulate phosphorylation of brush-border cell membranes of jejunum. Dr. Y. Shimonishi and colleagues have determined the amino acid sequences of three different STs isolated from ETEC and Yersinia enterocolitica and have elucidated the structureactivity relation of synthesized analogues of these STs. Shorter analogues of Yersinia ST have almost the same activity. The substitution of Glu-Leu in $E$. coli STs results in a loss of toxic activity, a finding suggesting that the strong toxicity of $E$. coli STs is due to either one of these two amino acid residues.

A monoclonal-antibody ELISA identifying STaproducing ETEC was found by Dr. M. R. Thompson to agree $100 \%$ with RIA and the suckling mouse assay. Dr. M. B. Cohen assessed ST binding and STinduced secretion on rat intestinal brush-border membranes and found that both processes are spontaneously reversible. Dr. Cohen suggested that various means of removing ST from its enterocyte receptor might be of therapeutic benefit. Finally, T. Honda discovered that $60 \%$ of $V$. cholerae non-O1 strains tested produce a new type of ST enterotoxin, as determined by amino acid sequence analysis. Dr. Honda also discovered that some non-O1 V. cholerae strains produce a hemolysin immunologically related to the ST produced by Vibrio parahaemolyticus. He hypothesized that this hemolysin may be responsible for the bloody diarrhea in some cases of infection with non-O1 $V$. cholerae.

\section{Immunology and Vaccine Development}

Iron-regulated outer-membrane proteins (IROMPS) that recognize and internalize the iron sideraphore are necessary for replication of $\boldsymbol{V}$. cholerae. Dr. R. A. Finkelstein reported that monoclonal antibodies to IROMPS were bactericidal for $V$. cholerae and bacteristatic for several gram-negative enteropathogenic bacilli. Dr. Finkelstein is searching for cross-reactive antibodies to IROMPS in human milk in an attempt to explain how human milk, obtained from mothers never exposed to $V$. cholerae, inhibits that organism.

Dr. J. D. Clements has engineered model, live oral vaccines for Salmonella and the $\boldsymbol{V}$. cholerae-ETEC diarrheas. He transformed attenuated Salmonella typhi strain Ty2la and an auxotropic Salmonella enteriditis strain, each with a recombinant plasmid coding for the nontoxic B subunit of $E$. coli LT. The derivative strains were avirulent, genetically stable, 
and antigenic for the gut mucosa of experimental animals. Dr. Clements is studying the potential contribution of other virulence antigens, such as ST toxoid and cholera somatic antigens, in the formulation of an optimum hybrid vaccine.

Dr. M. M. Levine summarized his group's attempts to develop less diarrheagenic, attenuated cholera vaccines. A series of genetically engineered strains of $V$. cholerae were tested in volunteers. So far, observations suggest that retaining the ability to colonize the proximal small intestine, in the hope of being able to immunize with a single dose of vaccine, leaves the strains inherently reactogenic. The goal is to strike a critical balance between satisfactory colonization and immunogenicity and tolerable reactogenicity. Dr. Levine has also studied ways to stimulate intestinal secretory IgA antibody to fimbriae (pili) adhesions in animals and humans. He used purified fimbriae or an attenuated non-ETEC strain as an oral vaccine. The results in volunteers point to the superiority of live oral vaccines, rather than purified fimbriae, in stimulating such protective antibody to fimbriae.

The superior antigenicity of live, rather than killed, $V$. cholerae fed to mice was confirmed by Dr. Y. Zinnaka. Living antigen induced delayed cutaneous hypersensitivity and serum antibody, whereas killed antigen induced serum antibody only. Forced feeding compared with ad libitum feeding suppressed serum antibody production, probably, according to the authors, by enhancing splenic suppressor-cell activity.

In the final paper on vaccines, Dr. J. Clemens summarized the progress of an ongoing field trial of two killed, oral cholera vaccines in Bangladesh. One vaccine contains B subunit and killed whole cells of V. cholerae, and the other vaccine contains killed $V$. cholerae cells alone. In early-phase studies on 1,257 volunteers, the two vaccinces were totally free of side effects; they induced a significant rise in vibriocidal antibodies or in antibodies to cholera toxin in $85 \%$ of vaccines after two doses. However, antibody titers were much lower than those after natural cholera or attenuated oral vaccines. The ability of the two vaccines to prevent cholera will be determined and reported in 1986.

\section{Pathophysiology}

Dr. S. C. Whipp found that in contrast to $E$. coli LT and STa, the pig-specific, mouse-negative $E$. coli toxin STb causes morphological damage to the pig jejunum and has functional correlates in reduced sucrose activity and interference with active transport of alanine. Dr. S. H. Richardson provided an update of his studies on the genetic and cellular control of the response of inbred mice to cholera toxin. Finally, A. Ghoda presented preliminary data suggesting that the house musk shrew, Suncus murinus, may provide a new laboratory animal model for $V$. cholerae and Shigella flexneri.

\section{Summary}

Cholera outbreaks with high death rates continue in countries lacking rehydration therapy. Strains of $E$. coli without identified $E$. coli virulence markers but adhering to HEp-2 cells likely represent a new class of enteropathogens causing travelers' diarrhea. Erythromycin is effective treatment for $C$. jejuni dysentery if the antibiotic is started early in the course of illness. Monoclonal antibodies have helped identify new virulence antigens on the outer membrane of $V$. cholerae. Several antigenically distinct Shiga and ST toxins have been discovered. Most EPEC strains contain a unique 94-kilodalton plasmid likely coding for a surface adhesion factor responsible for virulence. Activation of PKC in jejunal epithelial cells is associated with intestinal secretion. Genetically engineered, attenuated hybrid vaccines against $S$. typhi, V. cholerae, and ETEC have been successful in animal models and, in some cases, in volunteers. The goal of oral, attenuated enteric vaccines will be to strike a critical balance between satisfactory colonization and immunogenicity and tolerable reactogenicity. Meanwhile, two killed oral vaccines against $\boldsymbol{V}$. cholerae are being field tested in Bangladesh.

Dr. R. Bradley Sack, Chairman of the United States Cholera Panel, concluded that research on bacterial diarrhea is moving rapidly. Investigators must, however, (l) continue to investigate outbreaks as a way of finding new enteropathogens; (2) elucidate mechanisms producing bacterial toxin and antigen; (3) discover new bacterial virulence antigens for immunogens; and (4) improve therapy, vaccines, and rapid diagnostic tests.

The complete proceedings of the conference will be published by KTK Publishers (Tokyo). The next Joint Conference on Cholera and Cholera-Like Infections has been scheduled for July 1986 in Toyama City, Japan. 